\title{
Numerical Stability of a One-Evaluation Predictor-Corrector Algorithm for Numerical Solution of Ordinary Differential Equations
}

\author{
By R. W. Klopfenstein and R. S. Millman
}

1. Introduction. Predictor-corrector algorithms are often preferred over algorithms of the Runge-Kutta type for the numerical solution of ordinary differential equations, since the former may involve fewer derivative evaluations per forward step. As commonly implemented, forward values of the dependent variables are extrapolated by an open formula (the predictor formula) followed by an evaluation of the derivative expressions corresponding to the extrapolated values. This is followed by application of a closed formula (the corrector formula) to obtain new estimates of the dependent variables, and then a final evaluation of the derivative expressions is made corresponding to these new estimates. This step may be applied an arbitrary number of times, leading to a class of algorithms that may be denoted by $\mathrm{PE}(\mathrm{CE})^{n}$, where $n$ represents the number of applications of the corrector. The corrector may be iterated many times, but most commonly it is only applied once, since the improvement in truncation error through additional applications of the corrector is usually negligible.

A fourth-order PECE algorithm requires two derivative evaluations for each forward step, while a fourth-order Runge-Kutta algorithm requires four evaluations. Since the evaluation of derivatives typically dominates the total computation time in computer applications, this is considered a strong argument in favor of the use of predictor-corrector algorithms even though they require the storage of back values for the dependent variables and/or their derivatives, as well as requiring more involved procedures for interval modification. Proponents of RungeKutta methods often argue that these algorithms are "stable," while predictorcorrector methods may be "unstable" in varying degrees. Runge-Kutta methods, however, do have limits on their ranges of stability, while many predictor-corrector algorithms have regions of stability that compare favorably. The stability properties of predictor-corrector algorithms have been studied by a number of authors including Chase [1], Brown et al. [2], and Krogh [3]. In addition, Crane and Klopfenstein [4] have attempted to synthesize a fourth-order PECE algorithm having optimal stability properties, and have made comparisons with fourth-order RungeKutta methods. While judgments must be made that are difficult to quantize, there is much to be said in favor of using PECE algorithms in general-purpose algorithms for the solution of ordinary differential equations.

More recently, it has been suggested that an even further saving in derivative evaluations could be obtained by eliminating the final derivative evaluation in $\mathrm{PE}(\mathrm{CE})^{n}$ algorithms, thus obtaining a class of algorithms that may be denoted by $\mathrm{P}(\mathrm{EC})^{n},[5]$. For $n=1$, this results in a PEC algorithm requiring only one deriva-

Received July 17, 1967. Revised November 13, 1967. 
tive evaluation per forward step. While this is attractive from the standpoint of computation time, it may result in a substantial loss in stability. An experimental investigation by Hull and Creemer [6], confirmed by more recent studies of the characteristic equations [2], [3], has shown that when the fourth-order AdamsBashforth predictor is used with the fourth-order Adams-Moulton corrector in a PEC algorithm, the stable range is so reduced that the algorithm is virtually useless.

It is the object of the present work to explore the nature of the stability limitation for PEC algorithms. By employing methods previously used for PECE algorithms [4], an attempt is made to synthesize a predictor, for use with the fourthorder Adams-Moulton corrector, having optimal stability properties when implemented in a PEC mode. It will be seen that while substantial improvements can be made in the size of the stable region, the resulting algorithm must still be regarded as marginal when compared with existing PECE and Runge-Kutta algorithms.

2. The General Fourth-Order PEC Algorithm. The general PEC algorithm considered here for the numerical solution of

$$
y^{\prime}=f(x, y), \quad y(a)=y_{0},
$$

may be stated as

$$
\begin{aligned}
& p_{n+1}=a_{1} y_{n}+b_{1} y_{n-1}+c_{1} y_{n-2}+d_{1} y_{n-3}+h\left(e_{1} p_{n}^{\prime}+f_{1} p_{n-1}^{\prime}+g_{1} p_{n-2}^{\prime}+k_{1} p_{n-3}^{\prime}\right) \\
& y_{n+1}=a_{2} y_{n}+b_{2} y_{n-1}+c_{2} y_{n-2}+h\left(d_{2} p_{n+1}^{\prime}+e_{2} p_{n}^{\prime}+f_{2} p_{n-1}^{\prime}+g_{2} p_{n-2}^{\prime}\right)
\end{aligned}
$$

where $p_{n}{ }^{\prime}=f\left(x_{n}, p_{n}\right), \quad x_{n}=a+n h$, and appropriate starting values including $y(a)=y_{0}$ must be obtained by other means. We shall restrict our attention here to algorithms for which the predictor and corrector formulas are independently of fourth order, since this results in a truncation error for the combined algorithm that is identical to that for the corrector alone to first order [4]. This leaves three parameters in the predictor formula and two in the corrector that are available for improvement of numerical stability characteristics. These are arbitrarily selected to be $d_{1}, e_{1}, k_{1}, d_{2}$ and $e_{2}$. The relations expressing the remaining coefficients are given in [4].

There is much to be said in favor of specializing to the Adams-Moulton corrector at the outset. One is assured of some nonvanishing region of relative stability. Also, since the present study is directed at exploring the nature of the limitations of PEC algorithms, there is a good basis for comparison with results previously obtained [2], [6]. Specialization to the Adams-Moulton corrector fixes $d_{2}$ and $e_{2}$ as well as the rest of the corrector coefficients. The coefficients $d_{1}, e_{1}$, and $k_{1}$, henceforth referred to as simply $d, e$, and $k$, remain available for optimization purposes.

Therefore, the algorithm we will be concerned with is

$$
\begin{aligned}
& p_{n+1}=a y_{n}+b y_{n-1}+c y_{n-2}+d y_{n-3}+h\left(e p_{n}^{\prime}+f p_{n-1}^{\prime}+g p_{n-2}^{\prime}+k p_{n-3}^{\prime}\right), \\
& y_{n+1}=y_{n}+(h / 24)\left(9 p_{n+1}^{\prime}+19 p_{n}^{\prime}-5 p_{n-1}^{\prime}+p_{n-2}^{\prime}\right),
\end{aligned}
$$

with 


$$
\begin{aligned}
& a=9-d-3 e+3 k \\
& b=9-9 d+24 k \\
& c=-17+9 d+3 e-27 k, \\
& f=-18+6 d+4 e-17 k, \\
& g=-6+6 d+e-14 k .
\end{aligned}
$$

The characteristic equation corresponding to (2.3) may be found in the usual way $[1]$ and is given by

$$
\rho^{\overline{7}}+q_{6} \rho^{6}+q_{5} \rho^{5}+q_{4} \rho^{4}+q_{3} \rho^{3}+q_{2} \rho^{2}+q_{1} \rho+q_{0}=0,
$$

with

$$
\begin{aligned}
& q_{6}=-1+(\bar{h} / 8)(-27+3 d+e-9 k), \\
& q_{5}=(\bar{h} / 24)(180-44 d-15 e+135 k), \\
& q_{4}=(\bar{h} / 24)(-261+85 d+30 e-27 k), \\
& q_{3}=(\bar{h} / 24)(215-80 d-30 e+27 k), \\
& q_{2}=(\bar{h} / 24)(-94+35 d+15 e-135 k), \\
& q_{1}=(\bar{h} / 24)(17-4 d-3 e+27 k), \\
& q_{0}=(\bar{h} / 24)(-d),
\end{aligned}
$$

and $\bar{h}=h(\partial f / \partial y)$.

This characteristic polynomial is of seventh degree in $\rho$, but is linear in $\bar{h}$, corresponding to the single derivative evaluation performed for each step of the algorithm.

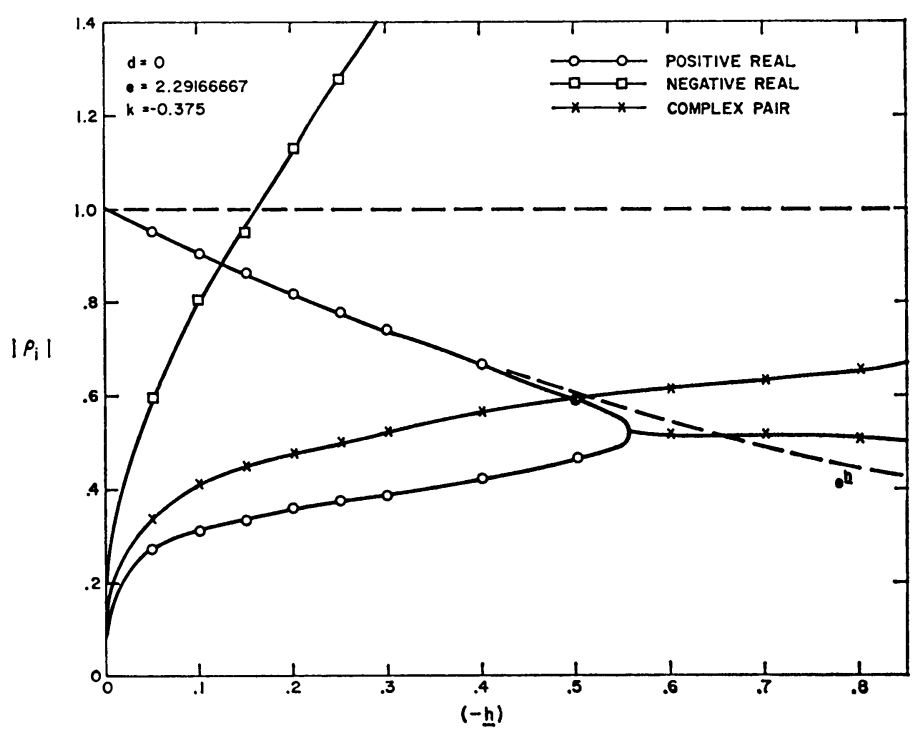

Figure 1. $\left|\rho_{i}\right|$ vs $(-\bar{h})$ for Adams-Bashforth/Adams-Moulton as PEC algorithm.

3. Numerical Stability Improvement. In order to improve the stability characteristics, we take the Adams-Bashforth/Adams-Moulton fourth-order algorithm as a starting point, i.e., $(d, e, k)=(0,55 / 24,-3 / 8)$. Fig. 1 exhibits the root loci of (2.4) as a function of real $h$, and is essentially a duplication of Fig. 1 in Brown 


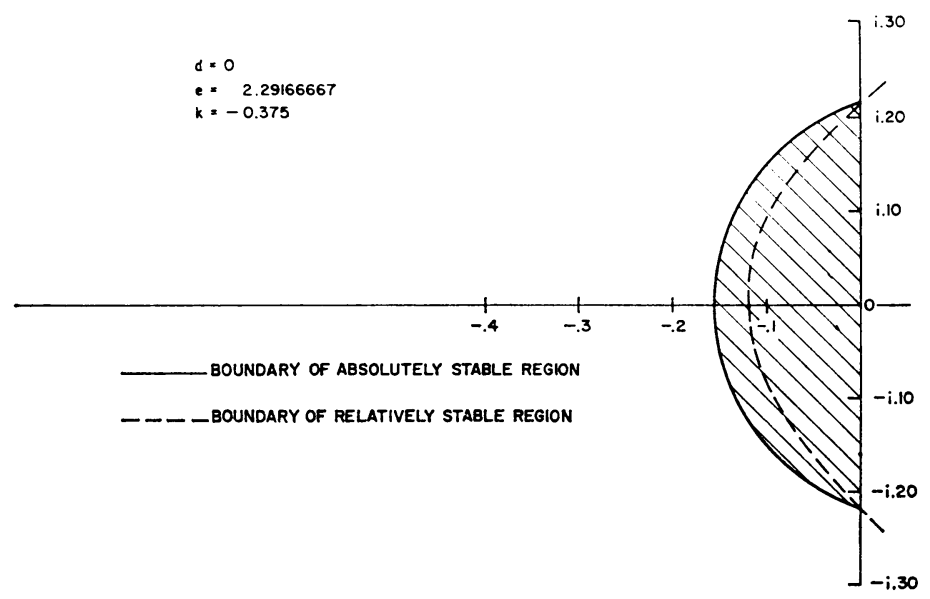

Figure 2. Region of stability in complex $\bar{h}$ plane for Adams-Bashforth/Adams-Moulton as PEC algorithm.

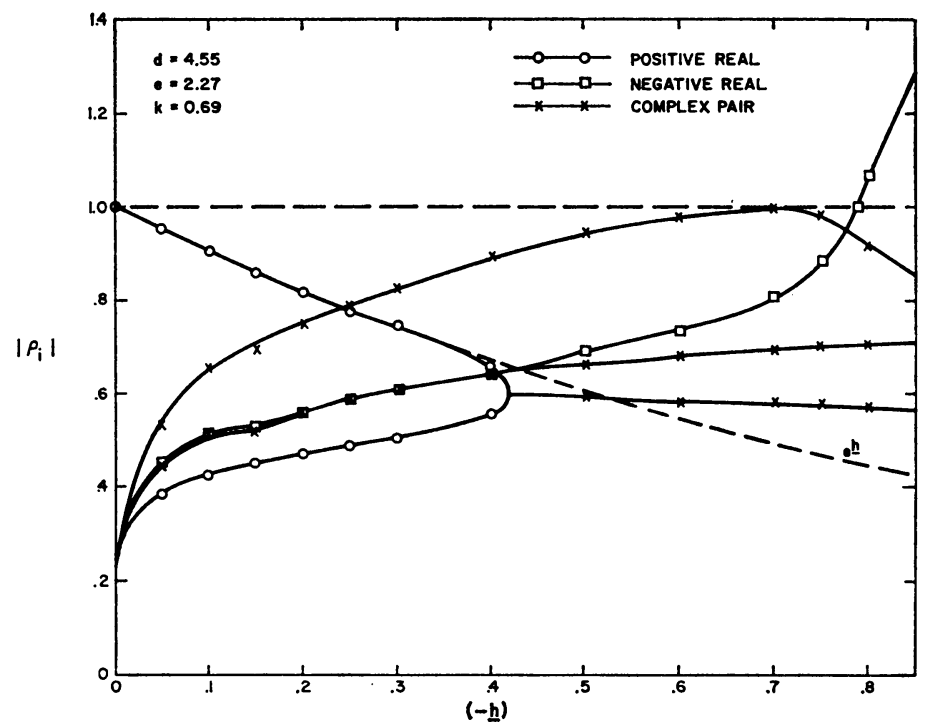

Figure 3. $\left|\rho_{i}\right|$ vs $(-\bar{h})$ for $K-M$ as PEC algorithm.

et al. [2]. Fig. 2 exhibits the boundary of the region in the complex $\bar{h}$ plane within which all roots of (2.4) are less than or equal to unity in absolute value. The region of absolute stability for a single differential equation or system of differential equations with real eigenvalues may be observed from Fig. 1. For systems of differential equations with complex eigenvalues, however, Fig. 2 must be consulted [4]. Dashed lines in each figure indicate the regions of relative stability for which all roots of (2.4) except the principal root (that one most nearly equal to $\exp (\bar{h})$ ) are equal to or less than $\exp ($ real part of $\bar{h}$ ) in absolute value. 


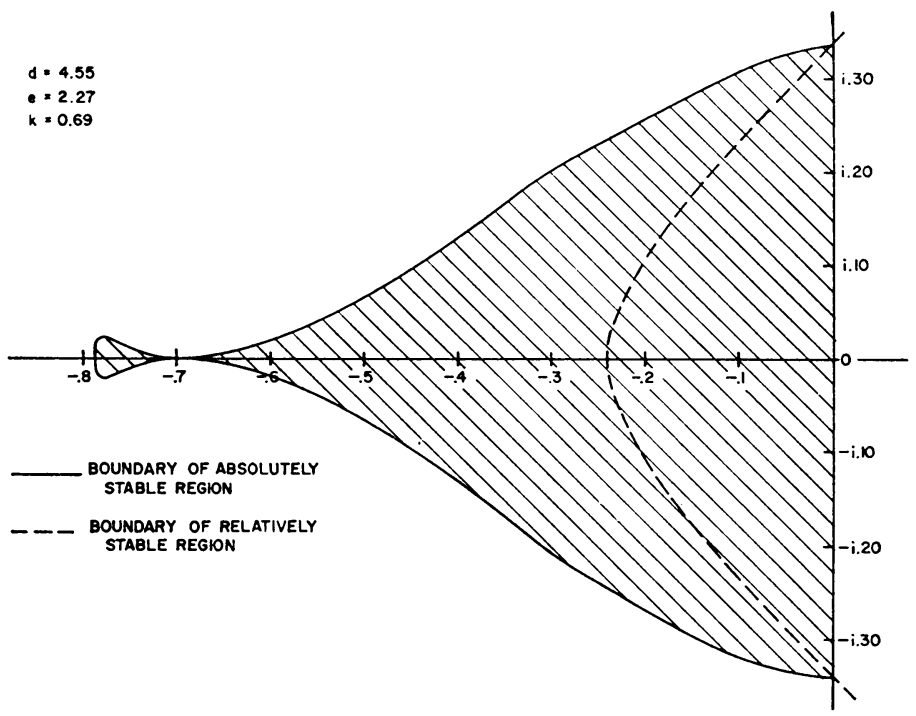

Figurl: 4. Region of stability in complex $h$ plane for $K-M$ as PEC algorithm.

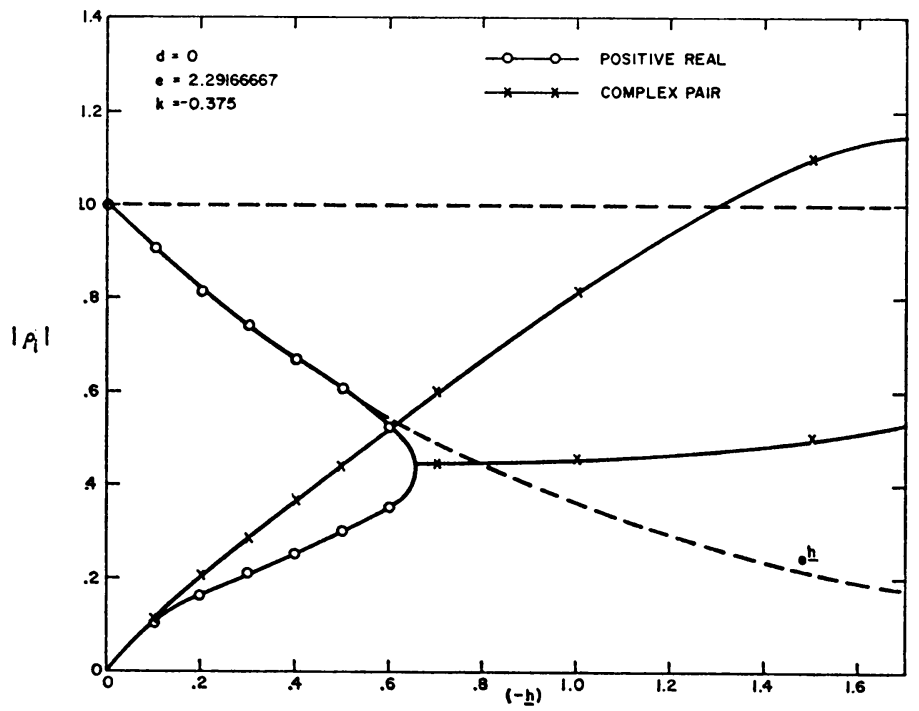

FigUre 5. $\left|\rho_{i}\right|$ vs $(-\bar{h})$ for Adams-Bashforth/Adams-Moulton as PECE algorithm.

For comparison, similar curves are given in Figs. 5 and 6 for the same formulas implemented in a PECE algorithm. These curves are plotted to half-scale to allow (visually) for the fact that twice as many derivative evaluations are required for each forward step. Even allowing for this, it can be seen that the PEC algorithm is markedly inferior to the PECE algorithm in stability characteristics. The stable region in Fig. 2 has about one-tenth the area of that in Fig. 6. This legislates strongly against the use of the formulas in a PEC algorithm. 


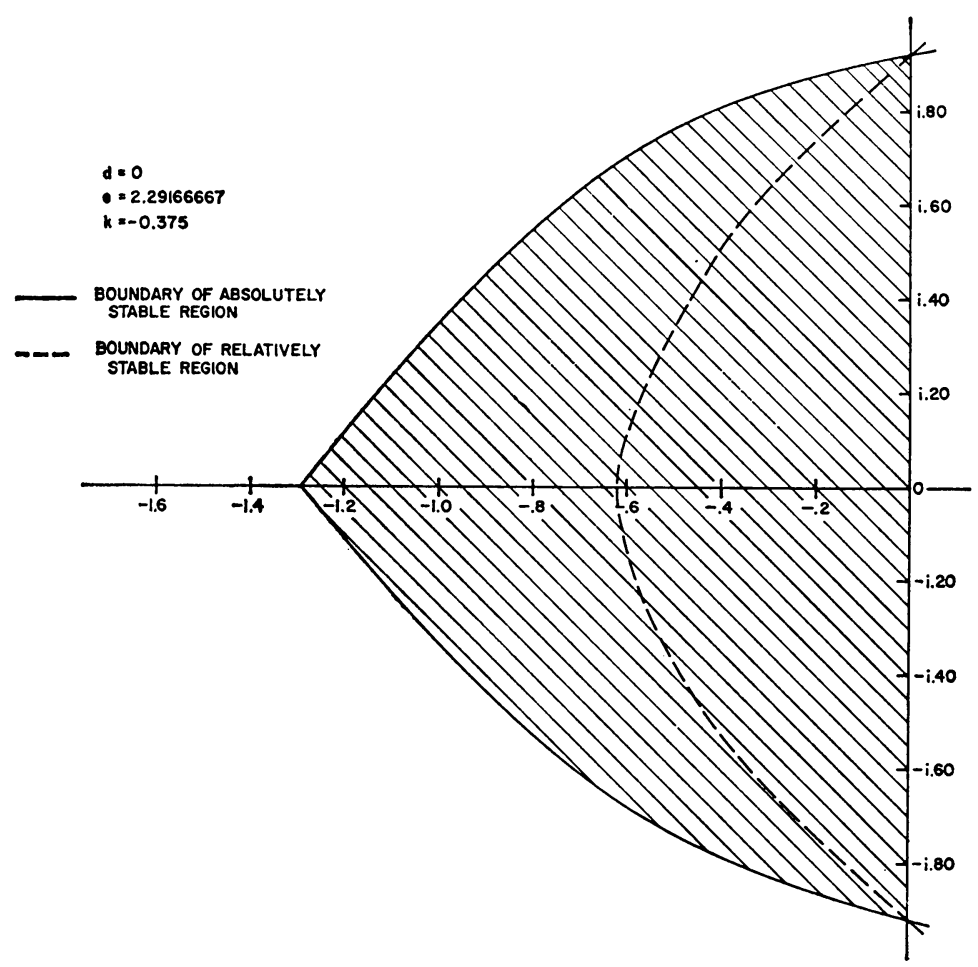

Figure 6. Region of stability in complex $\bar{h}$ plane for Adams-Bashforth/Adams-Moulton as PECE algorithm.

The process of improving the stability characteristics through synthesis of a new predictor formula makes use of the gradient technique described in [4] and will not be described in detail here. Some simplifications occur since the characteristic polynomial is linear in $\bar{h}$, but additional complications occur because of the increased degree in $\rho$ (seventh degree in place of fourth degree). Briefly, if one refers to the limiting value of $\bar{h}$ on the real plot (Fig. 1) as $\bar{h}^{*}$, the process consists of forming

$$
\operatorname{Grad}\left(\bar{h}^{*}\right)=\left.\left(\frac{\partial \bar{h}}{\partial d}, \frac{\partial \bar{h}}{\partial e},-\frac{\partial \bar{h}}{\partial k}\right)\right|_{\bar{h}=\bar{h}^{*}},
$$

in order to determine the direction in parameter space that will decrease $\bar{h}^{*}$ most rapidly. Evaluation of (3.1) requires implicit differentiation and depends on the nature of the limiting root (real positive, real negative, or complex pair of unit magnitude). One then proceeds in parameter space $((d, e, k)$-space) in the negative gradient direction with redeterminations of the gradient, as required, until a local extremum in $\hbar^{*}$ has been determined. While no assertion can be made that such a point represents an absolute extremum, it at least appears plausible that this is the best that can be done.

Applying the above procedure, a local extremum for $\bar{h}^{*}$ is found for the predictor in the form (2.3) with coefficients 


$$
\begin{aligned}
& a=-0.29, \\
& b=-15.39 \\
& c=12.13 \\
& d= \\
& e=2.55 \\
& f=2.27 \\
& f=6.65 \\
& g=13.91 \\
& k=0.69
\end{aligned}
$$

Since these coefficients satisfy the relations of (2.3), maximum significance is retained in the error estimator

$$
E_{p c} \cong \frac{p_{n}-y_{n}}{18.0274}
$$

which may be used to monitor the accuracy as an integration is carried forward by estimating the truncation error encurred at each step. In any adjustment of the coefficients of (3.2) (e.g., for different number bases) one should ensure that the relations of (2.3) are satisfied exactly to retain significance in (3.3).

The stability characteristics of the PEC algorithm given by (2.3), (3.2) are exhibited in Figs. 3 and 4 for real and complex $\bar{h}$. It is seen that substantial improvements in the size of the stable region have been obtained. On the real $\bar{h}$ axis, this PEC algorithm is absolutely stable for $-0.781 \leqq \bar{h} \leqq 0$, a range about five times that for the Adams-Bashforth/Adams-Moulton PEC algorithm. A respectably large region for complex $\bar{h}$ is obtained as well as reasonable relative stability characteristics. By and large, however, the stable region still lies within that for the Adams-Bashforth/Adams-Moulton PECE algorithm even after discounting the fact that only half as many derivative evaluations are required.

4. Numerical Computations. A number of computer runs were made comparing results obtained for first- and second-order linear differential equations using the PEC algorithm specified by (2.3), (3.2) with the results obtained using the AdamsBashforth/Adams-Moulton PECE algorithm. These runs verified the characteristics exhibited in Figs. 3-6. For smaller $\bar{h}$, the rounding-error accumulation of the PEC algorithm was larger by a factor of two or three than that of the PECE algorithm. This is thought to be due to the relatively large coefficients of the predictor (3.2), but was not judged to be a serious defect.

5. Conclusions. It has been suggested that computation time for predictorcorrector algorithms can be shortened by implementing them in a PEC mode, [5]. At least one commonly used predictor-corrector formula combination becomes so unstable in such an implementation as to be virtually useless [2], [6]. In the present study, we have explored the nature of this limitation of PEC algorithms by developing a predictor formula which, when used with the Adams-Moulton fourthorder corrector in a PEC algorithm, has improved stability characteristics. The size of the stable region for the resulting algorithm is still smaller than those for commonly used PECE algorithms. However, the new algorithm may be of interest in applications where stability is not a strong limitation. 
RCA Laboratories

Princeton, New Jersey 08540

1. P. E. Chase, "Stability properties of predictor-corrector methods for ordinary differential equations," J. Assoc. Comput. Mach., v. 9, 1962, pp. 457-468. MR 29 \#738.

2. R. R. BROWN, J. D. Riley \& M. M. BENNETT, "Stability properties of Adams-Moulton type methods," Math. Comp., v. 19, 1965, pp. 90-96. MR 31 \#2829.

3. F. T. KROGH, "Predictor-corrector methods of high order with improved stability characteristics,"J. Assoc. Comput. Mach., v. 13, 1966, pp. 374-385. MR 33 \#5127.

4. R. L. Crane \& R. W. KLopfenstein, "A predictor-corrector algorithm with an increased range of absolute stability," J. Assoc. Comput. Mach., v. 12, 1965, pp. 227-241. MR 31 \#6378.

5. M. A. Feldstein \& H. J. Stetter, Simplified Predictor-Corrector Methods, Assoc. Comput. Mach. National Conference, 1963.

6. T. E. Hull \& A. L. Creemer, "Efficiency of predictor-corrector procedures," J. Assoc. Comput. Mach., v. 10, 1963, pp. 291-301. MR $27 \# 436$ 7. 